\title{
VERIFICAÇÃO DO FATOR DE CALIBRAÇÃO E INDICADOR DA QUALIDADE DO FEIXE DE ACELERADORES LINEARES*
}

\author{
Maria da Penha Silva1, Helvécio Correa Mota², Carlos Eduardo V. de Almeida ${ }^{3}$
}

Resumo Um programa de garantia da qualidade é um pré-requisito obrigatório para a exatidão necessária em radioterapia. Este trabalho relata parte dos testes de rotina mensal do controle da qualidade dos aceleradores lineares do Instituto Nacional do Câncer, relativos à calibração dos feixes de fótons e elétrons, no período de dois anos. Os resultados foram comparados com as recomendações do protocolo AAPM TG-40. Na análise do fator de calibração para o feixe de fótons foi encontrado um desvio máximo de 12\%; para o feixe de elétrons o maior desvio encontrado foi $10 \%$. A flutuação observada no indicador da qualidade do feixe para os feixes de elétrons foi maior que para os feixes de fótons. Os resultados confirmam a importância de um programa de garantia da qualidade em um serviço de radioterapia, permitindo correções rápidas da dose administrada ao paciente.

Unitermos: Controle da qualidade. Fator de calibração. Indicador da qualidade dos feixes. Acelerador linear.

Abstract Verification of dose output and beam quality indicator in linear accelerators.

A quality assurance program is a mandatory prerequisite for obtaining the high level of accuracy required for radiotherapy. This paper reports the results of part of the routine quality control tests for linear accelerators at the National Cancer Institute, Brazil, performed monthly over a period of two years. These tests included dose output and beam quality indicator. The results were compared with the guidelines of the AAPM TG-40 protocol. The results for the photon beams have shown dose output variations of up to $12 \%$; for electron beams, the largest deviation found was $10 \%$. The fluctuations observed in the beam quality indicator for the electron beams were greater than for the photon beams. These results strongly emphasize the importance of a quality assurance program in radiotherapy services in order to allow prompt corrections of the dose delivered to the patient.

Key words: Quality control. Dose output. Beam quality indicator. Linear accelerator.

\section{INTRODUÇÃO}

No controle da qualidade de aceleradores lineares clínicos ${ }^{(\mathbf{1 - 4})}$, a verificação periódica da energia da radiação é uma tarefa árdua ${ }^{(5-7)}$.

Tais verificações são realizadas pelo menos mensalmente. Enquanto nos aceleradores lineares apenas uma ou duas energias de fótons são disponíveis, o número de energias de elétrons é freqüentemente em torno de seis a oito. As energias dos elétrons são tipicamente determinadas por meio de medidas do alcance dos elétrons ${ }^{(8-10)}$ e isto requer um

* Estudo desenvolvido como parte da Tese de Mestrado em Biociências Nucleares, Universidade do Estado do Rio de Janeiro (UERJ). Medidas realizadas no Serviço de Radioterapia do Instituto Nacional do Câncer (INCa), Rio de Janeiro, RJ.

1. Física do INCa, Ministério da Saúde.

2. Pesquisador do Instituto de Radioproteção e Dosimetria (IRD), Comissão Nacional de Energia Nuclear, Ministério de Ciência e Tecnologia.

3. Professor Titular em Física Médica do Laboratório de Ciências Radiológicas da UERJ, Físico do INCa.

Endereço para correspondência: Dra. Maria da Penha Silva. Rua Bom Pastor, 207, apto. 205, Tijuca. Rio de Janeiro, RJ, 20521-060. E-mail: mpenhas@hotmail.com

Aceito para publicação em 9/6/2001. fantoma de água para medidas de ionização na profundidade.

Um dispositivo confeccionado no Setor de Física Médica do Instituto Nacional do Câncer (INCa) foi utilizado para o controle mensal da qualidade dos aceleradores após a sua calibração com as condições de referência obtidas conforme as recomendações da Agência Internacional de Energia Atômica (IAEA International Atomic Energy Agency) $)^{(\mathbf{8 , 9})}$.

Neste artigo são mostrados a importância de um controle da qualidade para aceleradores lineares clínicos e o desempenho dos dois aceleradores existentes no Serviço de Radioterapia do INCa para os feixes de elétrons e fótons, com relação à calibração e ao indicador da qualidade do feixe de radiação.

\section{MATERIAL E MÉTODOS}

Uma câmara de ionização cilíndrica da Nuclear Enterprises, modelo NE2570/ $1 \mathrm{~B}$, foi utilizada nos feixes de fótons e elétrons com energias superiores a 10 $\mathrm{MeV}$, e uma câmara de ionização de pla- cas paralelas NACP foi utilizada para os feixes de elétrons com energias menores que $10 \mathrm{MeV}^{(4,10)}$.

As energias nominais de feixes de elétrons utilizadas foram de 6, 9, 13, 17 e $20 \mathrm{MeV}$ para o aparelho Therac 20, e 3, 6, 9 e $12 \mathrm{MeV}$ para o aparelho Saturne 1 e os feixes de fótons de 18 MV e 10 $\mathrm{MV}$, respectivamente.

Neste trabalho foi utilizado um fantoma de acrílico com dimensões de $30 \times$ $30 \times 30 \mathrm{~cm}$ para fornecer um espalhamento completo para o tamanho de campo medido $(10 \times 10 \mathrm{~cm})$. Foram utilizados também fantomas sólidos de polimetilmetacrilato (PMMA) nas medidas obtidas no dispositivo de teste (Figura 1) desenvolvido no Setor de Física Médica do INCa. O dispositivo é formado de duas hastes conectadas ao colimador do aparelho e que sustentam uma placa de madeira onde foram marcadas, no mesmo eixo, três posições para ser colocado o fantoma sólido de PMMA, de dimensão $10 \times 10 \mathrm{~cm}$, com profundidade variável de acordo com a energia dos feixes. Foram construídos dois fantomas sólidos: 


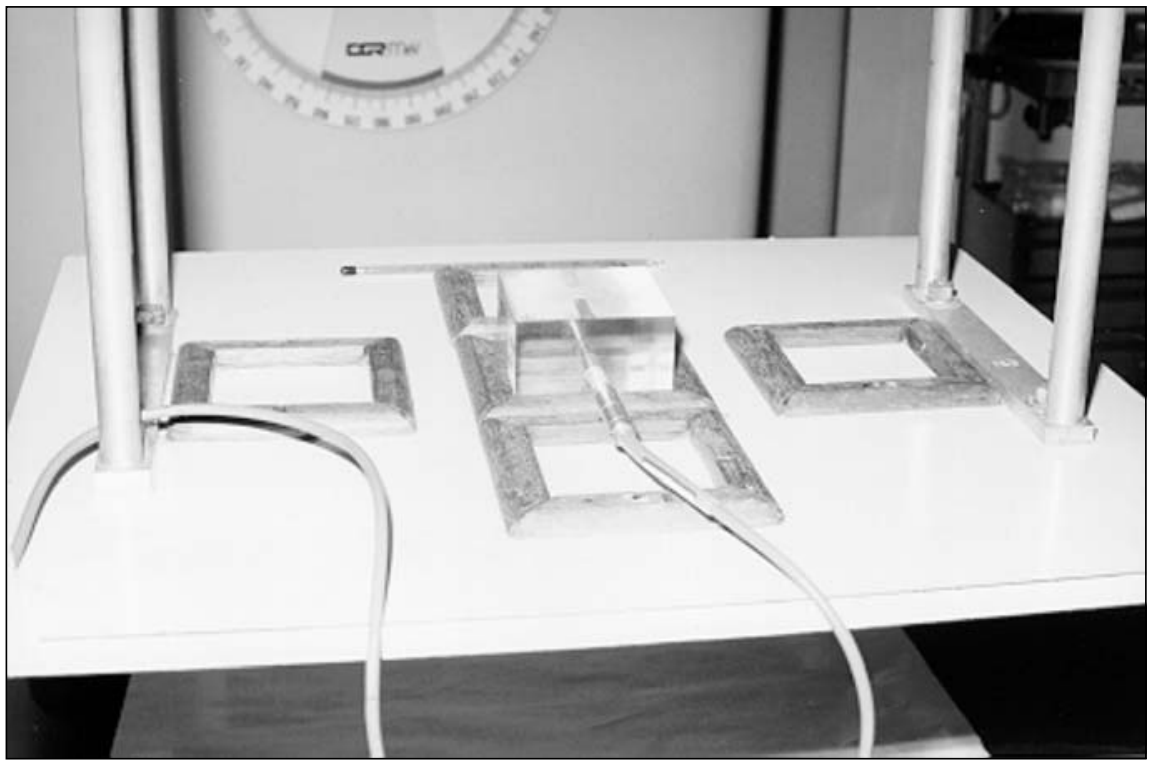

Figura 1. Dispositivo de teste.

um para o feixe de fótons e o outro para o feixe de elétrons. As posições escolhidas foram o eixo central do campo de radiação e dois limites, superior e inferior, deste campo. Foram utilizados os tamanhos de campo de $30 \times 30 \mathrm{~cm}$ para o feixe de elétrons e $40 \times 40 \mathrm{~cm}$ para o feixe de fótons. As medidas de calibração são obtidas na profundidade de equilíbrio eletrônico da energia em estudo. Para algumas energias torna-se necessário adicionar placas de PMMA ao fantoma sólido. Para as medidas do indicador da qualidade do feixe são obtidas as relações na profundidade de máximo e na profundidade correspondente a $50 \%$ do valor encontrado na profundidade de máximo. Placas de PMMA são adicionadas ao fantoma sólido, até obter a espessura desejada. Foram utilizadas medidas obtidas no dispositivo, com a finalidade de reduzir o tempo utilizado no controle da qualidade do acelerador linear.

Os fatores de calibração dos feixes de fótons e elétrons foram obtidos de acordo com o protocolo da IAEA ${ }^{(8)}$ para a distância de $1 \mathrm{~m}$. Os fatores de conversão do dispositivo de teste F (JIG) foram obtidos simultaneamente e considerados como valores de referência. As leituras foram igualadas e o fator de conversão do dispositivo para a água foi obtido utilizando a equação abaixo:

$$
\mathrm{D}(\mathrm{P} \text { eff })=\mathrm{L} \mathrm{Nd} \varnothing(\mathrm{P}, \mathrm{T}) \mathrm{F}(\mathrm{JIG})
$$

onde: L é a leitura média obtida no dispositivo; $\varnothing(\mathrm{P}, \mathrm{T})$ é o fator que corrige para temperatura e pressão; $\mathrm{Nd}$ é o fator de calibração do conjunto dosimétrico; D (P eff) é o valor da dose absorvida na água na calibração dos feixes.

Para o controle da qualidade da energia do feixe foram tomados como referência os valores obtidos na determinação do indicador da qualidade do feixe quando dos testes de aceite ${ }^{(9)}$.

\section{RESULTADOS}

Os valores dos indicadores da qualidade dos feixes de fótons e elétrons do aparelho Saturne 1, medidos no período de 20 meses e normalizados para os valores de referência, são encontrados na Figura 2. Para o feixe de fótons de 10 MV foi observada variação de $+7,5 \%$ e boa estabilidade no restante do período. Foi observada dispersão alta para o feixe de elétrons de $3 \mathrm{MeV}$, que é natural, por ser a menor energia de tratamento desse equipamento, sendo a maior variação encontrada de $+9 \%$. Para os feixes de 6 $\mathrm{MeV}$ e $12 \mathrm{MeV}$ existe tendência de aumento dessa variação, que deve ser corrigida. Para o feixe de elétrons de $9 \mathrm{MeV}$ existe a necessidade de reajuste do fator de indicador da qualidade do feixe. $\mathrm{Na}$ Figura 3, as flutuações encontradas, diferentes das recomendadas pelo protocolo da AAPM TG-40, foram reajustadas para o aparelho Therac 20. Observamos variações dentro da faixa de referência para o feixe de fótons. Para as energias de elétrons obtivemos variações de $+8 \%$ para a energia de $6 \mathrm{MeV}$ e $-8 \%$ para a energia de $17 \mathrm{MeV}$. Foram também observadas variações nas energias de $9 \mathrm{MeV}(+11 \%)$, $13 \mathrm{MeV}(+7,5 \%)$ e $20 \mathrm{MeV}(+5 \%)$.

Os valores obtidos de verificação do fator de calibração dos feixes foram normalizados para o valor do fator de calibração de referência e são mostrados na Figura 4 para o aparelho Saturne 1 e na Figura 5 para o aparelho Therac 20. Para o feixe de fótons de $10 \mathrm{MV}$ do aparelho Saturne 1, a maior variação encontrada no fator de calibração foi de $-12 \%$. Para os feixes de elétrons, os maiores desvios observados ocorreram nas energias de 3 $\mathrm{MeV}(-10 \%)$ e $12 \mathrm{MeV}(-8 \%)$; neste período, o valor do fator de calibração poderia ter sido renormalizado, conforme tendência observada no gráfico da Figura 4. Com relação ao equipamento Therac 20, o feixe de elétrons de $13 \mathrm{MeV}$ poderia também ter sido renormalizado, conforme tendência apresentada no gráfico da Figura 5; do mesmo modo, o fato ocorreu para o feixe de fótons de $18 \mathrm{MV}$. O maior desvio observado foi de $+7,5 \%$ para a energia de $20 \mathrm{MeV}$. A variação deste fator reflete diretamente na dose administrada ao paciente.

\section{CONCLUSÕES}

O método descrito para verificar a constância das energias dos feixes de elétrons e fótons num acelerador linear, baseado na intercomparação das medidas realizadas num fantoma de água e no dispositivo de PMMA, é conveniente.

A verificação diária do fator de calibração dos feixes de fótons é recomendada em vários protocolos, a fim de garantir que os valores permanecerão dentro da faixa de tolerância para esses aparelhos. Para assegurar o tratamento do paciente com o mesmo padrão de qualidade, as medidas diárias são importantes, pois qualquer variação que possa ocorrer com o equipamento será logo detectada e serão tomadas as providências para solucionar a alteração. 


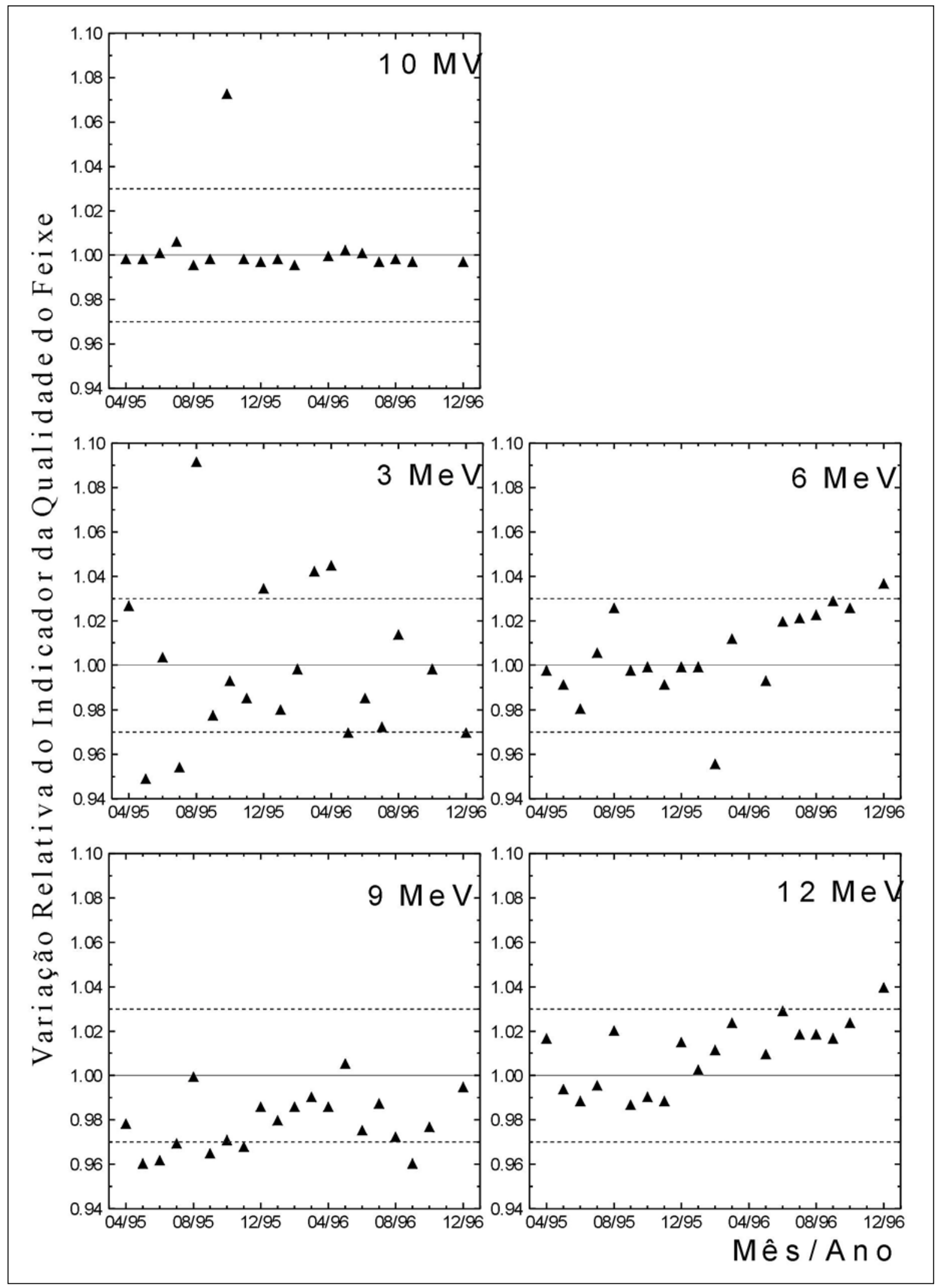

Figura 2. Variação relativa do indicador de qualidade dos feixes de fótons (10 MV) e elétrons $(3,6,9$ e $12 \mathrm{MeV})$ do Saturne 1 , normalizado para o valor de referência. As retas pontilhadas representam a variação aceitável, conforme o programa de garantia da qualidade no INCa. 


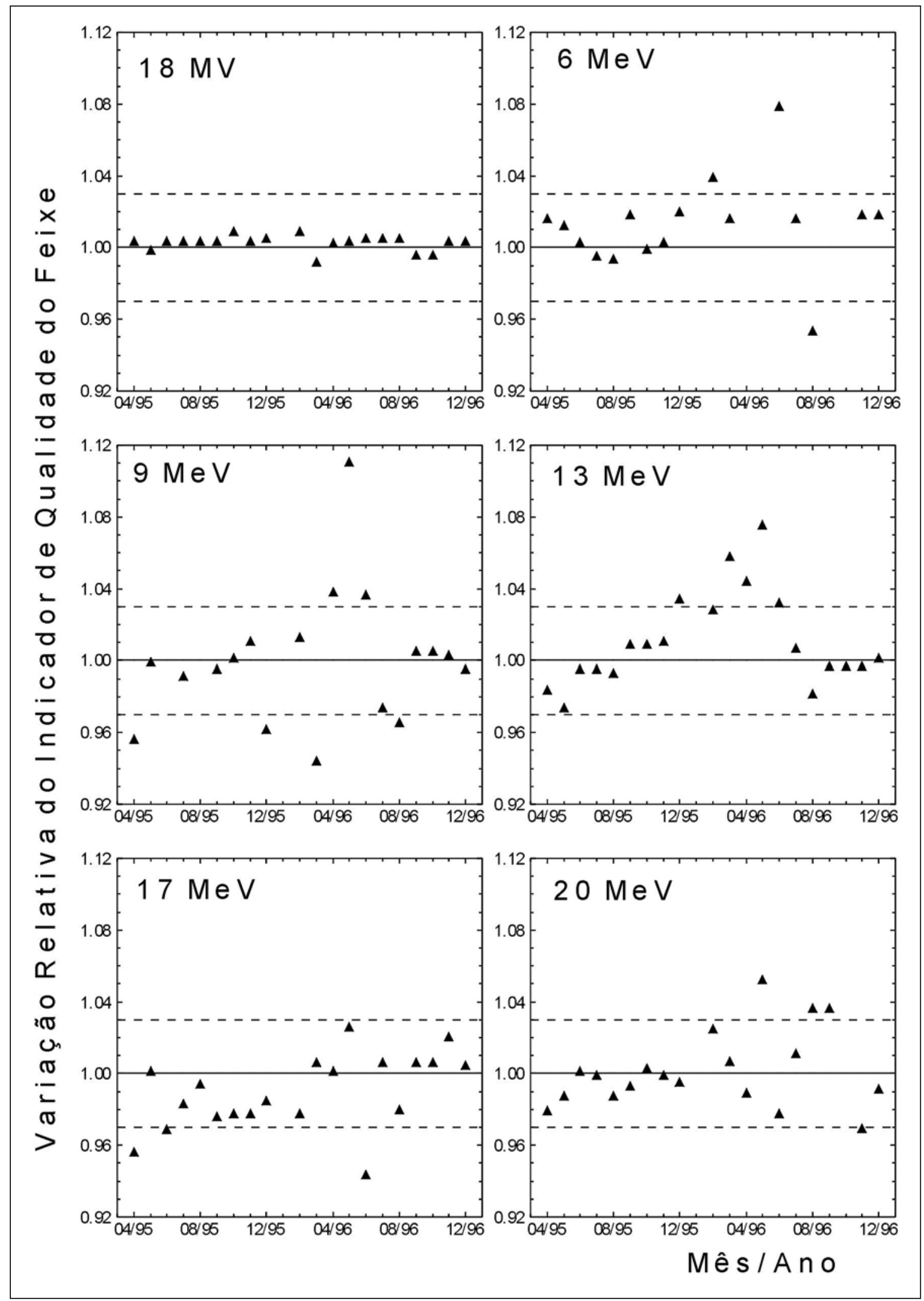

Figura 3. Variação relativa do indicador de qualidade dos feixes de fótons (18 MV) e elétrons (6, 9, 13, 17 e $20 \mathrm{MeV}$ ) do Therac 20, normalizado para 0 valor de referência. As retas pontilhadas representam a variação aceitável, conforme o programa de garantia da qualidade no INCa. 


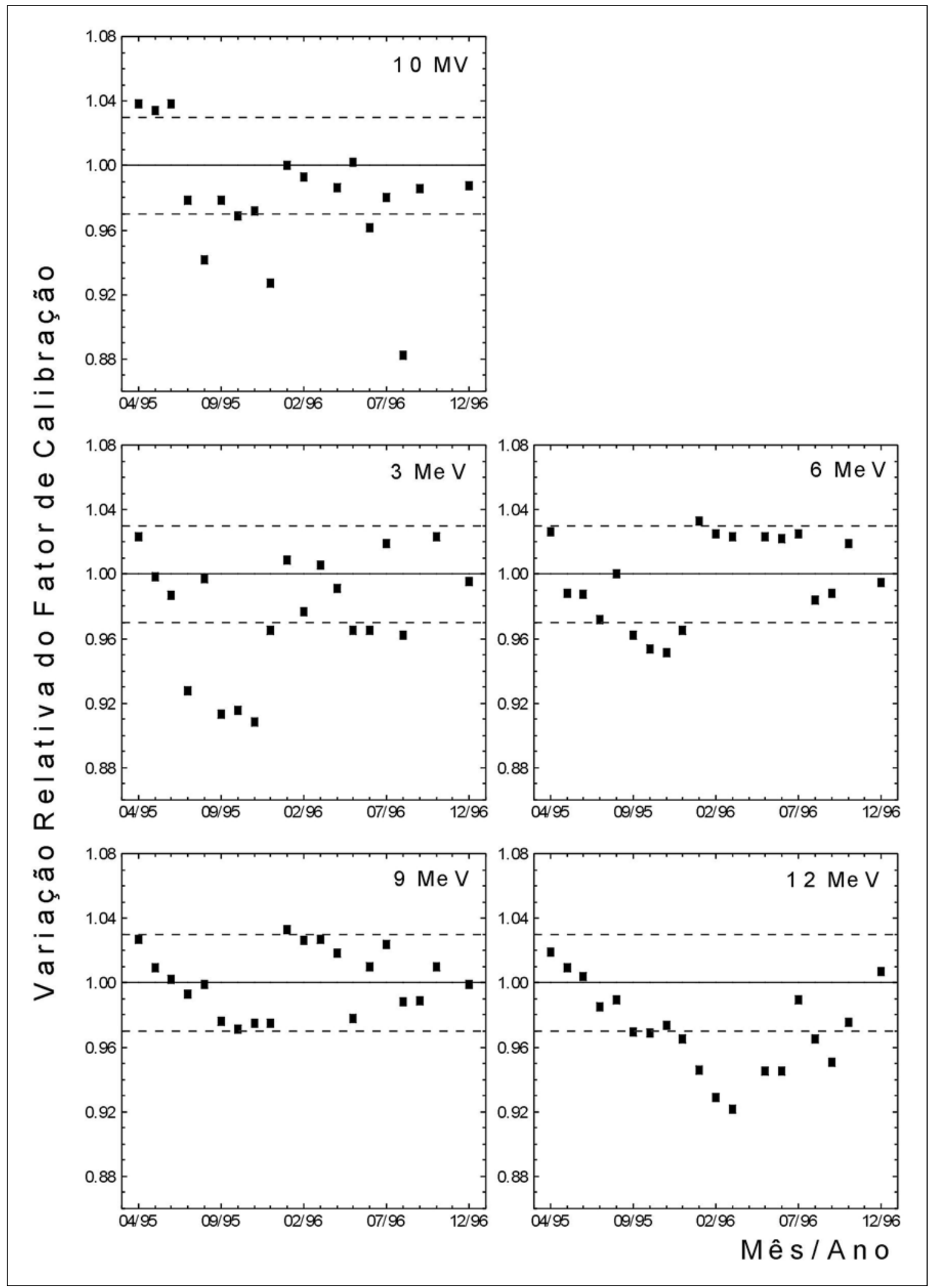

Figura 4. Variação relativa do fator de calibração dos feixes de fótons (10 MV) e elétrons $(3,6,9$ e $12 \mathrm{MeV})$ do Saturne 1, normalizado para o fator de calibração de referência. As linhas pontilhadas representam a variação aceitável, conforme o programa de garantia da qualidade no INCa. 


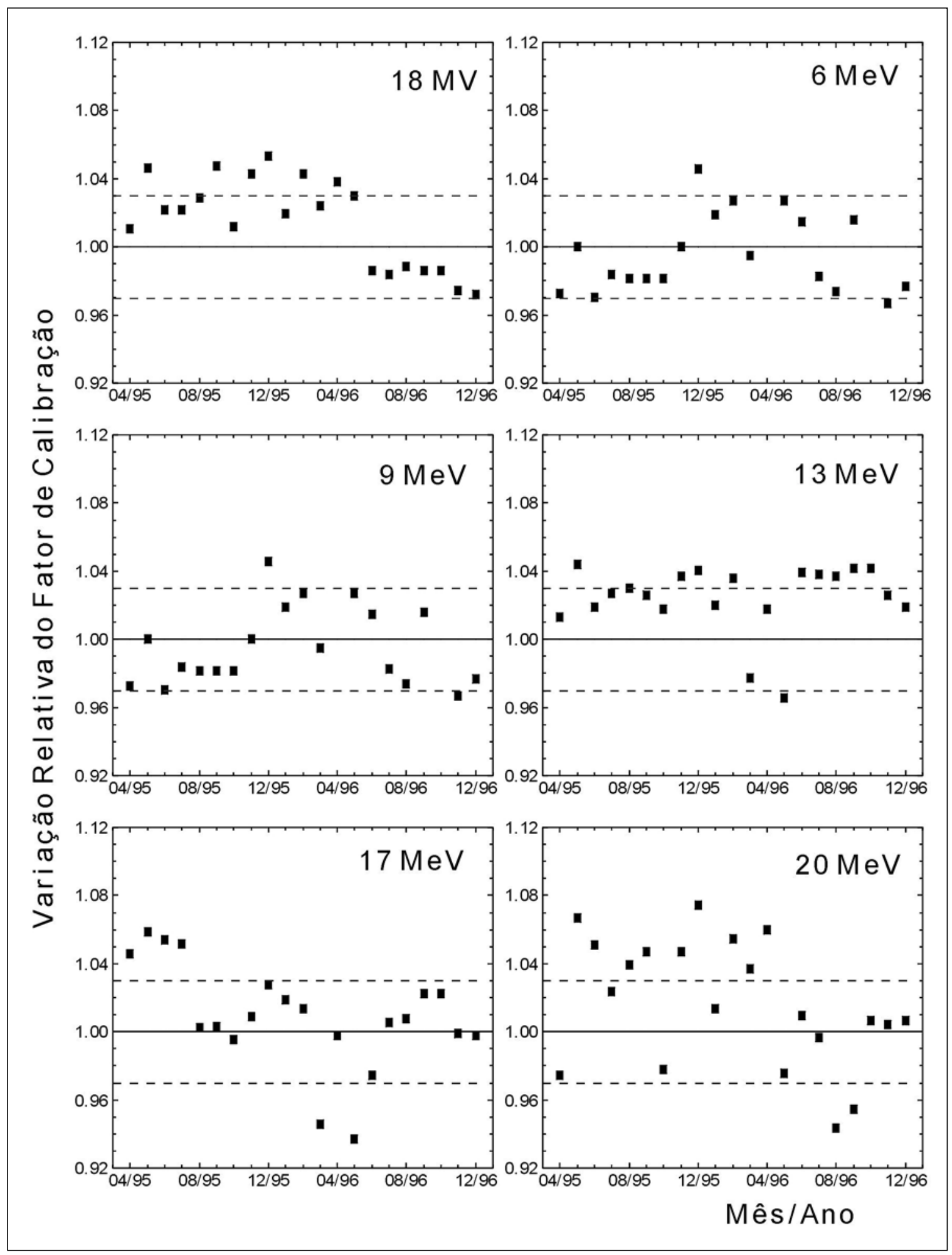

Figura 5. Variação relativa do fator de calibração dos feixes de fótons (18 MV) e elétrons (6, 9, 13, 17 e $20 \mathrm{MeV})$ do Therac 20, normalizado para o fator de calibração de referência. As linhas pontilhadas representam a variação aceitável, conforme o programa de garantia da qualidade no INCa. 


\section{REFERÊNCIAS}

1. Associação Brasileira de Físicos Médicos. Protocolo para controle de qualidade em unidades de megavoltagem em radioterapia. São Paulo: ABFM, 1995.

2. Dutreix A, Mijnheer B, Svensson H. New protocols for the dosimetry of high-energy photon and electron beams. Radiother Oncol 1985;4:289-90.

3. Khan FM. Quality assurance. In: The physics of radiation therapy. 2 nd ed. Baltimore: Williams \& Wilkins, 1992:504-42.

4. Dutreix A, Derreumaux S, Chavaudra J, van der
Schueren E. Quality control of radiotherapy centres in Europe: beam calibration. Radiother Oncol 1994; 32:256-64.

5. American Association of Physicists in Medicine. A protocol for the determination of absorbed dose from high energy photon and electron beam. New York: AAPM, Task Group 30, 1983.

6. Kutcher GJ, Coia L, Gillin M, et al. Comprehensive QA for radiation oncology: report of AAPM Radiation Therapy Committee Task Group 40. Med Phys 1994;21:581-618.

7. Nath R, Biggs PJ, Bova FJ, et al. AAPM code of practice for radiotherapy accelerators: report of
AAPM Radiation Therapy Task Group No. 45. Med Phys 1994;21:1093-121.

8. International Atomic Energy Agency. Absorbed dose determination in photon and electron beams. Viena: IAEA, Report 277, 1987.

9. International Atomic Energy Agency. Calibration of dosimeters used in radiotherapy. Viena: IAEA, Report 374, 1994.

10. International Commission on Radiation Units and Measurements. Radiation dosimetry electron beams with energies between 1 and $50 \mathrm{MeV}$. ICRU, Washington Report 35, 1984. 